\title{
Reflets
}

Revue d'intervention sociale et communautaire

\section{Mesures et indicateurs de la performance en travail social : l'impossible évaluation des pratiques professionnelles}

\section{Entrevue avec Vincent Meyer}

\section{Stéphane Richard}

Volume 19, numéro 2, automne 2013

La souffrance psychique et morale au travail : enjeux pour les professionnels du secteur de la santé et des services sociaux

URI : https://id.erudit.org/iderudit/1021178ar

DOI : https://doi.org/10.7202/1021178ar

Aller au sommaire du numéro

Éditeur(s)

Reflets, Revue d'intervention sociale et communautaire

ISSN

1203-4576 (imprimé)

1712-8498 (numérique)

Découvrir la revue

Citer ce document

Richard, S. (2013). Mesures et indicateurs de la performance en travail social :

l'impossible évaluation des pratiques professionnelles : entrevue avec Vincent

Meyer. Reflets, 19(2), 26-42. https://doi.org/10.7202/1021178ar 


\section{Mesures et indicateurs de la performance en travail social : l'impossible évaluation des pratiques professionnelles}

\section{Entrevue avec Vincent Meyer}

Vincent Meyer est professeur à l'Université de Nice Sophia Antipolis en France et membre du laboratoire Information Milieux, Médias, Médiations (I3M). Il siège au Conseil supérieur du travail social (CSTS) où il préside la sous-commission Veille et propositions sur des sujets d'actualité liés aux pratiques, aux enjeux territoriaux et à l'impact des thématiques émergentes sur le travail social.

L'entrevue a été réalisée par Stéphane Richard.

Reflets: Un des concepts que vous avez développés dès les tout débuts de votre carrière universitaire est celui des "communications d'action et d'utilité publiques ", pouvez-vous nous l'expliquer? Et constitue-t-il la clé de voûte des multiples domaines d'intérêt que vous avez développés par la suite?

V. Meyer : D'abord, il importe de préciser que pour moi, les « communications d'action et d'utilité publiques » réferent à ce qu'on peut appeler une notion, ce n'est pas un concept. C'est une notion que j'ai stabilisée fin 2004. Au départ, mon travail s'est caractérisé par une étude de la communication dans le social, dans l'intervention sociale; c'est le travail que j'ai réalisé dans le cadre de ma thèse.J'ai étudié un double mouvement. D'un côté, ce qui est médiatisable dans le travail social et de l'autre, la part du social qui revient - dans les années 1980 et 1990 - aux médias. Cet ensemble caractérise 
ce que j’ai appelé l'« émergence du sociomédiatique ». Après cette réflexion sur le sociomédiatique, j'ai engagé une réflexion sur les usages des équipements médiatiques autour des nouvelles technologies de l'information et de la communication. Ce qui a donné naissance à une autre notion, celle de "sociotique ".

C'est aussi en 2004 que j'ai soutenu mon habilitation à diriger les recherches en sciences de l'information et de la communication sur l'étude de différentes campagnes de prévention, de sensibilisation, des opérations de recueil de dons, mais aussi tout le développement d'une réflexion méthodologique sur la manière dont il faut travailler ces différentes formes de communication publique. En fait, les communications d'action et d'utilité publiques, c'est la notion qui vient, pour le moment, clore toute une réflexion sur la manière dont on communique dans le social, la manière dont les médias et les institutions publiques s'emparent des questions sanitaires et sociales pour communiquer.

Reflets: En quelque sorte, ce n'est pas nécessairement la toile de fond de toutes vos réflexions autour de la thématique de Reflets qui porte notamment sur la souffrance au travail et les façons d'y faire-face chez les travailleuses et travailleurs sociaux.

V. Meyer: Non, c'est davantage du côté des travaux du Groupe national des établissements publics sociaux et médicaux-sociaux (GEPSo) que j'ai abordé ce sujet. En sachant que finalement, ces questions de souffrance au travail peuvent aussi entrer dans des opérations de sensibilisation, des campagnes de prévention liées à différents mondes professionnels.

Reflets: Qu'est-ce qui vous a donc amené à vous intéresser au travail social et aux personnes qui s'impliquent dans ce champ d'étude et d'intervention?

V. Meyer: C'est une question à la fois simple et très complexe parce que dans une période particulièrement douloureuse de ma vie, j'ai dû arrêter mes études et je suis entré par la petite porte dans le monde du travail social. J'ai commencé ma carrière comme moniteur-éducateur, puis j'ai été éducateur spécialisé avant de faire le diplôme supérieur du travail social et de rédiger une thèse de sociologie pour intégrer l'université. 
Reflets: C'est donc la pratique qui vous a mené à réfléchir et à poursuivre vos études et recherches?

V. Meyer : En effet, j'ai commencé à travailler dans un établissement d'accueil pour personnes handicapées mentales, à l'époque, un institut médicoprofessionnel. J'ai commencé ce travail en mars 1981. Je n'avais absolument aucune formation dans le social. Je ne savais pas ce qu'était une personne handicapée. Je ne savais même pas ce qu'était un travailleur social; et la première question que je me suis posée à ce moment-là était : que faut-il comme compétence pour intervenir auprès de ces personnes? Et, croyez-le bien, cette question n'a cessé de structurer mon travail de recherche.

J'ai une formation de travailleur social,je suis un travailleur social diplômé, mais pendant toute cette période-là, je n'avais qu'une idée : reprendre mes études que j'avais dû arrêter à un moment de ma vie. Tout en travaillant, je suis retourné à l'université pour y préparer d'abord une maîtrise, ensuite un diplôme d'études approfondies et ensuite un doctorat. J'ai travaillé dans plusieurs établissements. J'ai donc été travailleur social pendant près de quinze ans avec différentes responsabilités sur le terrain.

Reflets : Est-ce que vous êtes principalement intervenu auprès des clientèles en déficience intellectuelle ou physique?

V. Meyer : Bien que dans des stages j'aie eu affaire à des personnes en difficulté sociale, c'est quand même essentiellement le monde du handicap mental et surtout sa prise en charge institutionnelle que j'ai connus. Et, c'est ce qui a aussi guidé l'ouvrage qui est paru sur la communication organisationnelle et le handicap mental. C'est une réflexion ethnographique sur la manière dont on communique dans les milieux clos au sens goffmalien du terme, dans ce qui était encore des institutions totales... ou qui le sont parfois encore à des degrés divers.

Donc, si vous voulez, et c'est ce que me disent un certain nombre de collègues, je n'ai jamais quitté le travail social; le travail social est toujours resté une base de recherche. Et autant j'étais praticien chercheur à un moment donné, autant maintenant je suis devenu 
un chercheur qui s'intéresse à toutes les questions qui touchent de près ou de loin le travail social.Voilà pourquoi aussi je pense que j'ai été nommé au Conseil supérieur du travail social (CSTS) pour assurer différentes fonctions et présidences en son sein.

Reflets : Pourriez-vous nous parler de votre implication au Conseil supérieur $d u$ travail social (CSTS) en France?

V. Meyer : Le Conseil supérieur du travail social est structuré en différentes commissions. La première est la Commission du Rapport. Elle vient de présenter début 2013 à la ministre un rapport sur le partage d'informations. Ensuite, il y a une Commission Éthique et Déontologie qui réfléchit sur un certain nombre de questions éthiques posées en travail social. Il y a enfin la Sous-commission Veille qui porte sur les activités et les thématiques émergentes dans le travail social. Moi,j'ai la chance de présider cette dernière dans laquelle nous avons mis sur Internet une plate-forme de veille, un moteur qui nous permet d'avoir un certain nombre d'alertes via des flux RSS. Nous réalisons également des auditions et des notes problématiques : les deux dernières concernent la performance dans le travail social, l'intervention sociale auprès des 18-25 ans et les difficultés que rencontrent cette population et les travailleuses et travailleurs sociaux qui la prennent en charge.

Reflets : Est-ce que la Sous-commission Veille est reliée aux deux autres commissions que vous avez identifiées?

V. Meyer: Bien sûr, nous travaillons ensemble et nous nous alimentons réciproquement pour qu'il y ait à la fois un partage d'informations et aussi parce que toutes les questions, celles liées à la performance, par exemple, ont une dimension éthique. Il y a donc de facto des travaux conjoints. Le CSTS est là aussi pour capter les besoins et les demandes des travailleuses et travailleurs sociaux et pour être un relais vers les instances ministérielles.

Reflets : En lien avec les travaux de la Sous-commission Veille, vous abordiez vos études et travaux sur la question de la performance dans le travail social. Peut-on lier ces travaux avec les réalisations et réflexions du GEPSo et votre implication à la direction des quatre numéros suivants publiés 
par Les Études Hospitalières : celui de 2004 (Éthique et mémoire), de 2008 (Les usagers évaluateurs), de 2010 (Normes et Normalisation en travail social), de 2012 (Performance, sens et usure dans les pratiques des professionnels en travail social)?

V. Meyer: Pour ce qui concerne la note que nous avons produite pour la Sous-commission Veille du CSTS, initialement, il n'y avait pas de lien avec les travaux du GEPSo, dans la mesure où c'est le CSTS qui a décidé des thématiques. Et la question de la performance s'est presque naturellement imposée à nous avec les agences qui ont été créées en France, notamment l'Agence nationale d'appui à la performance. Cette notion est quelque chose de très problématique pour le terrain; nous nous sommes donc emparés de la question. Au GEPSo, elle a été traitée lors du dernier séminaire que nous avons organisé en 2012. Avec le CSTS, nous avons rencontré des organisations syndicales et réalisé des auditions auprès de différents responsables pour avoir une vision la plus large possible de ce que pose aujourd'hui comme questions cette performance en travail social. Il est extrêmement important de rester en contact avec le terrain et d'être aussi le relais d'un certain nombre de préoccupations directes des travailleuses et travailleurs sociaux. Sur ce point, bien sûr, la notion de performance interroge fortement ces derniers par sa définition, par ses objectifs et surtout par sa mesure.

On a également rencontré des acteurs du terrain qui s'interrogent beaucoup sur les possibles dérives et sur les effets pervers de ce type de démarche, parce qu'ils ont la crainte qu'elle soit largement instrumentalisée ou parfois mal dimensionnée. Donc, nous avons d'abord commencé à réfléchir sur le lien entre l'évaluation et la performance et voir comment aujourd'hui, les pratiques d'évaluation intègrent cette notion de performance.

Ensuite, nous avons réfléchi sur tout ce qui est mesures et indicateurs, c'est-à-dire, comment construit-on aujourd'hui un certain nombre d'indicateurs? Est-ce que c'est dans une logique de tableau de bord partagé? Est-ce que c'est uniquement des indicateurs de différents niveaux de pilotage? Comment se construit aujourd'hui une mesure des indicateurs dans le travail 
social? Parce que finalement, on nous dit que refuser aujourd'hui de rentrer dans cette démarche de performance, c'est être dans un déni complet des réalités économiques. La saine gestion des deniers publics imposerait à tous maintenant d'être dans ces différents indicateurs et mesures. On pensait qu'avec le changement de gouvernement en France les choses allaient bouger un peu. On n'est, dès lors, pas persuadé que les choses vont suffisamment dans le bon sens...

C'est aussi pour mettre à mal tout ce discours qui dit que le travail social ne peut pas être en dehors du monde réel. On ne peut pas être dans une relation enchantée. Il faut quand même qu'il y ait des résultats, des indicateurs, etc., et qu'enfin, on nous dise qui fait quoi et comment dans ce social. Or, ce qu'on a relevé sur le terrain, c'est qu'aucune mesure ne s'est encore imposée à ce jour et qu'il demeure extrêmement difficile de définir des indicateurs pour, par exemple, mesurer l'accompagnement des personnes, pour se situer face à l'usager. Alors bien sûr, on nous dit qu'il faut des référentiels pour étalonner les pratiques, mais en même temps, comment, par exemple, établir des outils de mesure de la participation à l'action d'un usager où à une action d'accompagnement?

Cette notion empruntée au modèle marchand et productif peut avoir des effets désastreux sur la manière dont on pense les prises en charge, mais aussi sur des logiques de concurrence entre les différents établissements. Elle est souvent pensée comme une rationalisation des interventions dont le seul objectif est une diminution des coûts. C'est un des glissements que nous avons repérés au CSTS.

Un autre glissement, c'est la question du sens. Et là, ça rejoint effectivement le travail du Comité scientifique du GEPSo; parce que finalement, à trop vouloir mesurer ou être dans des successions d'indicateurs, il y a un risque de perte de sens. Paradoxalement, une recherche très poussée de performance peut éloigner les travailleuses et travailleurs sociaux du sens même de leur action et peut-être réduire l'efficacité d'un dispositif de prise en charge. Pour forcer le trait, on peut dire qu'ils vont être plus occupés à mesurer leur performance qu'à être dans une relation à l'autre, dans 
des objectifs de travail qui doivent être non pas en contradiction, mais en adéquation avec ce qu'on leur a demandé de faire et ce qui est important de faire pour garder un niveau de performance versus un niveau de financement.

Pour nourrir cette réflexion, nous disposons maintenant des quatre ouvrages du GEPSo publiés par son Comité scientifique de l'éthique des pratiques professionnelles et de l'évaluation (CSEPPE). Dès sa création au début des années 90, le CSEPPE s'est engagé dans une réflexion sur l'évaluation dans les établissements sociaux et médico-sociaux. Le GEPSo est essentiellement une association du secteur public et du secteur institutionnel en France. La première publication, liée à Éthique et mémoire, visait à réfléchir le " déjà-là ", la question posée était la suivante : fallait-il attendre le développement législatif récent pour penser l'évaluation? Autrement dit, comment, s'est construite - ou non - une mémoire des pratiques en même temps qu'une éthique professionnelle? On peut avancer sans trop se tromper que depuis la loi de 1975, et pour être plus précis, depuis le développement des établissements sociaux et médico-sociaux, il existe une réflexion éthique et une réflexion sur les pratiques.

Rappelons que dans les établissements sociaux et médico-sociaux, il y a des trésors en termes d'archives sonores, vidéos, photographiques qui témoigneraient de la façon dont les personnes ont été prises en charge. On a là un corpus extrêmement précieux qui n'est pas encore exploité.Voilà pourquoi nous a vons travaillé en 2004 cette dimension pour en fin de compte dire qu'avant de penser "l'évaluation " dans l'actualité, il fallait voir comment elle avait évolué dans les établissements par le passé.

Chaque fois, le GEPSo lie la sortie d'un ouvrage à un séminaire qu'il organise et où sont conviés les auteurs et aussi des conférenciers et des praticiens. C'est aussi un point fort du GEPSo : cette dimension de confrontation entre les pratiques de terrain et la réflexion sur le plan académique et universitaire et cela sans que l'une ait le primat sur l'autre. On est pour ainsi dire dans une coconstruction du sens.Voilà pourquoi ces ouvrages ont une portée véritablement interdisciplinaire au sens le plus strict du 
terme; c'est-à-dire qu'il y a des professionnels et des universitaires qui essaient de réfléchir, d'écrire et de travailler ensemble sur certaines thématiques.

Reflets: Ce qui est intéressant dans cette logique du GEPSo, c'est qu'il y a une évolution de la pensée, une pensée qui est toujours arrimée avec la pratique. Parlez-nous maintenant, en ce sens, de l'ouvrage Les usagers évaluateurs et des autres volumes subséquents.

V. Meyer: En effet, l'ouvrage Les usagers évaluateurs vise à situer la place que l'on donne à l'usager dans l'évaluation? Actuellement en France, la question de la participation des usagers à l'évaluation des pratiques est posée d'une manière très frontale. Nous, on a déjà pris cette réflexion à bras le corps un peu plus tôt en disant : mais comment, aujourd'hui, pouvons-nous nous priver de cette parole de l'usager dans la manière dont il va évaluer les pratiques de prise en charge?

Il existe un certain nombre de pratiques; il y a toute une histoire des établissements et la loi est venue renforcer, développer ces pratiques d'évaluation et a posé l'usager au centre de ce dispositif. Il faut donc voir de près comment les choses se font. Est-ce que l'usager est simplement un faire-valoir? Est-ce qu'il participe véritablement? Et s'il participe, quels sont véritablement les apports de ce type de participation dans l'évaluation?

À partir de là s'est posée la question des normes et des normalisations. Aujourd'hui, on aimerait que dans le travail social, il puisse y avoir des pratiques un peu plus normées, que ce ne soit pas cette espèce de grand malstrom de pratiques hétérogènes d'une institution à une autre.Voilà pourquoi dans Normes et normalisations, il y avait un sous-titre : Pour une posture critique entre responsabilité, résistance et créativité. Peut-être faut-il vraiment résister aux normes et affirmer qu'on est en permanence dans une logique de création plutôt que dans une logique normative dans ces pratiques.

Ensuite s'est posée la question de la performance. Et dans le dernier ouvrage, on l'a très directement associée - et c'est un geste à la fois scientifique et politique - à l'usure dans les pratiques 
professionnelles. Pour nous, cette question de la performance renvoie bien à celle du sens du et au travail, mais aussi à la question des souffrances dans ce dernier et, au-delà peut-être, à une réflexion sur la compétence des individus dans l'exercice de leur pratique. À dire vrai, toutes ces questions m'ont interpellé depuis le premier jour où j'ai mis les pieds dans un établissement social. Qui a la compétence de dire qui est compétent dans le social?

Je me souviens d'un ancien chef de service qui me disait qu'à partir du moment où tu racontes que c'est bien pour l'usager, tu peux avoir une paix royale. En fonction de cela,je me suis toujours interrogé sur la manière dont on produit de l'information sur l'usager, la manière dont on traduit sa situation, ses mal-être, ses bien-être, les situations dans lesquelles il se trouve. Cela renvoie immanquablement à cette question de l'évaluation, à sa mise en mots, à sa mise en indicateurs, à sa mise en situation et c'est cet ensemble-là que nous avons travaillé aussi avec ces questions de performance.

Bien évidemment, c'est lié aussi à l'actualité du travail social, à l'existence d'une Agence nationale d'appui à la performance, à l'existence aujourd'hui de toutes les recommandations de notre Agence nationale d'évaluation. On ne sait pas trop, avec les nouvelles lois, ce que vont devenir ces agences-là.Vont-elles être fusionnées? Vont-elles garder leur autonomie? Dans tous les cas, c'est cette évolution-là que nous avons voulu montrer au travers de ces quatre numéros du GEPSo.

Reflets : En lien avec le présent numéro de Reflets qui porte essentiellement sur ce qui cause la souffrance, ses effets et les moyens ou façons d'y faire face chez les professionnels, $j$ 'aimerais vous entendre sur les quelques pistes $d u$ faire-face développées par des auteurs des numéros de 2010 et de 2012 autour de la résistance et de la créativité des travailleuses et travailleurs sociaux.

V. Meyer: En fait, le reproche, c'est que cette créativité serait une espèce d'écran de fumée; finalement, que crée-t-on de nouveau dans une relation à l'autre? La rhétorique de la créativité ne serait 
peut-être là que pour masquer l'incompétence des travailleuses et travailleurs sociaux à rétablir les situations, à être dans les logiques des médiations et à réaliser véritablement un travail d'accompagnement.

En ce sens, je crois qu'actuellement la question de la compétence des travailleuses et travailleurs sociaux est posée. Je pense aussi que c'est quelque chose qui entraîne de la souffrance parce que, en permanence, ils sont dans une logique de justification de leur pratique. Et plus les dispositifs d'évaluation se développent, plus ils sont dans cette logique de justification de leur pratique. Et là, je dois quand même dire que c'est un peu de leur faute, parce que peut-être n'ont-ils pas vu venir le coup? Ont-ils toujours été inféodés à différentes instances, que ce soit du côté de la psychologie? De celui de la médecine, à un moment donné? La recherche sur les pratiques en travail social est encore à développer, bien que nous ayons au Conservatoire national des arts et des métiers une chaire de Travail social et d'intervention sociale avec un doctorat. Avec elle, les pratiques gagneraient en légitimité au lieu d'être en permanence menacées. Les travailleuses et travailleurs sociaux sont dans une logique permanente d'incertitude qui est pour eux très difficile à vivre, me semble-t-il.

Reflets : Dans l'introduction de Normes et normalisation en travail social, vous souleviez l'idée que les dispositifs d'évaluation organisationnels peuvent dans les faits figer l'engagement professionnel des travailleuses et travailleurs sociaux, leur pratique, bref, qu'ils peuvent laisser peu de place au savoir acquis dans l'expérience, à la créativité, à l'innovation. Pourriez-vous nous en parler davantage?

V. Meyer: J'ai tendance à dire que c'est de leur faute, alors qu'ils ont été largement inféodés aux sociologues, aux psychologues, aux chercheurs. Ces derniers aussi se sont largement professionnalisés dans le champ du travail social. Quand je vois la tonne de mémoires qui traînent dans les instituts de formation en travail social, quand je vois le peu de recherches-actions qui sont menées. Il semble ne pas y avoir de dynamique qui permettrait aujourd'hui aux travailleuses et travailleurs sociaux de devenir ce que Patrick Boumard (1989) a joliment appelé « les savants de leur propre 
réalité ». Ça, c'est une des grandes difficultés, s'ils ne veulent pas rester de simples exécutants de politiques publiques décidées en dehors d'eux.

N'y voyez absolument aucune forme de liens de cause à effet, mais c'est vrai que mon interrogation de départ sur la compétence n'a pas encore trouvé de réponses à ce jour. Parce que lorsque je suis entré dans le travail social, il y a eu une socialisation qui s'est réalisée dans l'interaction avec l'usager, dans l'interaction avec les collègues; et on s'est aperçu qu'il n'y avait pas vraiment de pratiques stabilisées. Il y avait peut-être des formes de savoirfaire qui étaient liées à un certain nombre de conduites, mais des pratiques, il n'y en avait pas; d'où la distinction que je fais entre conduite et pratique professionnelles. Souvent, je dis qu'il n'existe pas de pratique professionnelle dans le travail social, mais une succession de conduites. Qu'est-ce à dire? Les conduites, pour moi, c'est à la fois des dimensions très routinières dans une prise en charge, des actes qu'on fait un peu mécaniquement; ou alors, effectivement, c'est dans ce qu'on appelle des situations de crise ou de tension où il faut réagir par rapport à une urgence ou par rapport à une situation particulièrement difficile pour l'usager ou pour le travailleur social. La pratique finalement, ça serait un certain nombre de conduites qui seraient agrégées, mais qui seraient toujours dans une logique de justification; c'est-à-dire, que la pratique vient toujours pour se justifier et non pas pour montrer les compétences que peuvent avoir les personnes en situation de travail et les résultats qu'elles peuvent être amenées à produire par la prise en charge qu'elles mettent en œuvre. Et je fais ainsi une distinction entre conduite et pratique; et je me dis qu'actuellement encore, dans le travail social en France - et c'est peut-être différent chez vous - on parle beaucoup de pratique, mais je crois que si actuellement nos agences parlent de référentiels, de la normalisation, c'est que ces pratiques-là ne sont pas encore tout à fait stabilisées.

Je pense que les travailleuses et travailleurs sociaux ont une vraie responsabilité là-dedans. 
Reflets : Vos propos m'amènent à vouloir vous entendre davantage sur les contributeurs aux travaux du GEPSo, et plus particulièrement ceux qui cherchent à susciter la résistance critique des professionnels face à l'évolution actuelle de la généralisation des procédures d'évaluation au travail (culture de performance); face à cette évolution susceptible de moduler les activités professionnelles des travailleuses et travailleurs sociaux et surtout leur lien avec les usagers des services.

V. Meyer: À propos de ceux qui ont contribué aux travaux du GEPSo, disons d'abord qu'ils sont choisis pour leur esprit critique. Nous avons notamment des personnes comme Michel Chauvière qui, je pense, est connu au Canada et qui a une position très critique par rapport au développement actuel de la marchandisation du social. Rappelons que ce qu'on essaie de faire au travers du GEPSo et de son Comité scientifique, c'est de rassembler autour de ces différentes thématiques des personnes qui ont vraiment une vision critique, pas des béni-oui-oui qui essaient de ramer dans le sens du courant, et se dire voilà, nous allons fédérer autour de ces thématiques un certain nombre de chercheurs dont on sait que la position est critique. Ça, c'est la première dimension. Ensuite, ce qu'on essaie de faire, c'est d'amener à réfléchir sur les travailleuses et travailleurs sociaux. C'est-à-dire que les ouvrages, on l'espère bien, ont une vie après leur parution et qu'ils peuvent nourrir un certain nombre de travaux sur les activités professionnelles en tant que tel. Donc, la dimension critique est dans le choix que nous avons des conférenciers, un choix extrêmement ciblé et parfois aussi très politique. Et de l'autre côté, on a cet espoir que ces différentes contributions vont pouvoir animer dans les équipes de terrain une réflexion sur les activités professionnelles des travailleuses et travailleurs sociaux. C'est surtout ça qu'on a essayé de mettre en place. La résistance critique, elle se fait à la fois par l'appel à des penseurs et par le fait que ces travaux-là peuvent déclencher un certain nombre de réflexions sur des situations de travail dans les établissements et dans les services.

Bref, je dois vous avouer que pour le moment, on n'a pas encore réussi à mesurer comment nos réflexions sont reprises par le terrain et que je ne sais pas, pour reprendre une métaphore un 
peu générale, si on a été très performant en ce sens. On a parfois l'impression que la réflexion s'arrête aux travaux que nous menons, mais qu'on n'arrive pas tellement à contaminer le terrain avec pareille réflexion. Bref, je suis un peu inquiet, car je trouve que les travailleuses et travailleurs sociaux ne sont plus vraiment en résistance.

Reflets : Au fait, comment préserver chez ces professionnels cette motivation pour le travail bien fait? L'exercice du jugement professionnel?

V. Meyer: Les professionnels que nous avons rencontrés dans les différentes auditions ont tous véritablement témoigné de leur motivation à exercer dans le travail social. On sent très bien qu'il y a, du moins chez les professionnels que nous avons rencontrés, qui étaient aussi parfois des porte-parole d'organisations syndicales et autres, un véritable engagement et une véritable implication dans le travail. Mais en même temps, il y avait cette crainte d'être dessaisis du sens de ces pratiques au travers de cette normalisation qui avance progressivement. Et je pense que la motivation, tel que vous l'abordez dans cette question, c'est peut-être avant tout une valorisation de ce qui se fait. On avait l'impression que l'action des travailleuses et travailleurs sociaux était en permanence dénigrée, très peu valorisée, et que cette situation est assez difficile à vivre pour eux.

En 2014, nous allons vivre les États généraux du travail social en France dans lesquels cette question de la motivation, de la valorisation et de l'image des travailleuses et travailleurs sociaux dans l'espace public va être posée d'une manière très directe. C'est aussi toute la question liée à l'image dans l'espace public et cela renvoie aux travaux que j'ai menés en 2004, au livre qui s'intitule Interventions sociales, communication et média. L'émergence du sociomédiatique.

Reflets: Est-ce que la méthode des scénarios offre justement un lieu de débat visant à problématiser cette situation de tension (normative) vécue par les travailleuses et travailleurs sociaux dont nous parlons?

V. Meyer: La méthode des scénarios, c'est quelque chose qu'on a essayé de développer à Metz et qui l'a souvent été dans le cadre du travail 
social, par Jean-Yves Trépos notamment. Moi, je l'ai utilisée aussi pour travailler certaines situations. La méthode des scénarios, c'est une méthodologie projective et participative appliquée à l'enquête en sciences humaines et sociales. Elle s'applique particulièrement bien au développement de recherche-action. La particularité de la méthode des scénarios est justement de jouer sur les justifications, parce que vous savez - pour le dire avec un peu d'humour - il y trois façons d'obtenir de l'information des gens. La première c'est la méthode coercitive : on vous pose un flingue sur la tempe et on vous dit qu'on a les moyens pour vous faire parler, une méthode qui existe depuis la nuit des temps pour obtenir de l'information les uns et des autres. Il y a également des formes d'empathie, c'est-à-dire que vous avez un rapport à l'autre qui fait qu'il va vous donner des informations. Et vous avez la troisième voie qui est celle de la justification. Parce que finalement, on ne donne jamais autant d'information sur ces pratiques professionnelles que lorsqu'on est obligé de les justifier. Et la méthode des scénarios vise justement par sa mise en œuvre à produire des justifications, à ne pas rester dans le discours très lisse et très légitime qu'on peut obtenir lorsqu'on travaille par entretien semi-directif dans lequel par exemple on produit souvent du discours autorisé et légitimé sur l'institution et sur les pratiques. On va essayer ainsi d'aller un peu au-delà et de provoquer grâce à cette méthode-là un certain nombre de justifications qui vont éclairer sur la manière dont les personnes parlent de leurs pratiques, les justifient à titre individuel et dans un collectif.

Reflets : Cette méthode offre donc un lieu de débat qui pourrait problématiser la tension des travailleuses et des travailleurs sociaux. C'est aussi un débat qui pourrait éventuellement impliquer les dirigeants, ceux qui décident des normativités ou de ce que doivent faire les professionnels.

V. Meyer : Complètement, parce que normalement il y a une phase individuelle où l'on demande aux personnes de se projeter dans différents scénarios qui sont construits. Par exemple, Jean-Yves Trépos les a utilisés pour travailler avec des assistantes sociales. Il a construit des scénarios. Il a fait dire dans ces scénarios ce que jamais une assistante sociale ne dirait à voix haute sur un usager. 
Les participantes réagissent ainsi par écrit sur ces scénarios produits par le chercheur. Ensuite, il y a une première analyse de contenu qui est réalisée; puis, on réunit ces personnes dans un débat collectif et on leur dit : "Voici les éléments que vous avez produits autour de ce scénario et maintenant, nous allons en discuter.» Et très vite, on entre dans les justifications parce qu'on ne veut pas laisser dire un certain nombre de choses sur ces conduites professionnelles. Et on est dans une logique de justification de ces différentes pratiques. Mais ce n'est pas une justification de type individuelle, c'est aussi une justification qui est régulée par le groupe. Donc, il $\mathrm{y}$ a des choses que l'on ne va pas laisser dire, mais il y a d'autres qu'on va dire. Oui, bon! C'est vrai! Normalement, on doit traiter tous les usagers de la même façon. Mais de temps en temps, il nous arrive quand même de faire ci, de faire ça. On essaye avec cette méthode des scénarios de recueillir des représentations de premier plan, mais aussi des représentations de second plan sur les conduites professionnelles.

Reflets: Cette méthode de justification en groupe peut-elle ainsi permettre l'élaboration d'un jugement critique et nous placer sur la voie de l'autonomie professionnelle?

V. Meyer: Normalement, la méthode de scénarios est utilisée dans de l'investigation sociologique classique, mais on doit la développer aussi dans le cadre de la recherche-action. Celle-ci reste une coproduction de sens dans laquelle le chercheur ne vient pas avec une position surplombante imposer son savoir. Il travaille avec le savoir expert produit par les personnes en situation. Et dans la partie débat, généralement à la fin, on doit arriver à ce qu'on appelle un accord sur un certain nombre de situations, c'est-à-dire que les questions peuvent être effectivement liées à la souffrance, à la manière dont on peut $\mathrm{y}$ faire face, à la manière dont on va se positionner par rapport à tel ou tel cas ou telle situation de personne dans l'établissement. On est aussi dans une aide à la décision et dans ce que vous appelez dans votre question peutêtre l'élaboration d'un jugement critique. Mais pas un jugement critique qui est individuel, plutôt quelque chose qui est réfléchi dans une dynamique de groupe spécifique que les scénarios vont 
pousser à construire. Ce qui est encore différent des focus groups que vous pratiquez aussi au Canada.

Reflets: Cette méthode, par la notion de réappropriation du sens, permet donc de se réapproprier le sens, de dire les choses et d'accéder à un jugement critique à travers le justificatif?

V. Meyer : C'est quelque chose qui marche très bien, parce que les personnes savent que finalement, ce sont des scénarios, que c'est le chercheur qui les a construits, que ce sont des artéfacts. Ils se disent, par exemple : «Je ne peux pas laisser dire ça sur ma profession! ", «Je ne peux pas laisser produire ce type de discours! », «Je dois réagir,je dois trouver les mots parce que les scénarios sont tellement tordus que je dois les retordre dans l'autre sens! » Ça, c'est une dimension sur laquelle on a essayé de travailler; et cette méthodologie projective et participative fonctionne particulièrement bien parce que dans la première partie, on demande aux personnes de réagir individuellement et par écrit. Parfois, on leur laisse de 8 à 15 jours pour réagir. Nous, ce qui nous intéresse, c'est qu'ils nous rendent le matériau avec lequel ils sont le plus en accord possible. Vous savez, il n'est pas question ici de répondre à un questionnaire en 5 minutes, avoir un entretien semi-directif en 45 minutes. On laisse plutôt le temps aux personnes de rentrer dans les scénarios, de travailler et de nous produire une version avec laquelle ils sont les plus satisfaits possible. Ensuite, nous réalisons une première analyse de contenu et nous revenons vers eux.

Reflets : En terminant, et à la lumière de vos propos sur la souffrance au travail, ce qui la cause, ses effets et les façons d'y faire face, quels seraient les angles de recherche à prioriser en travail social à l'avenir?

V. Meyer: Alors, je crois qu'il y a deux pistes : la valorisation du travail social dans un premier temps. Je pense qu'il y a vraiment une question aujourd'hui qui est liée à l'image des travailleuses et travailleurs sociaux dans l'espace public. Dans un second temps, il y a vraiment la constitution d'une praxis, c'est-àdire, d'une réflexion qui se fasse par le terrain sur les pratiques professionnelles. Et je crois qu'à partir du moment où on prend en compte la difficulté ou la véritable souffrance au travail liée, 
par exemple, au fait de travailler avec des personnes lourdement handicapées, ça entraîne une forme de souffrance. Donc, de savoir que ce travail-là peut être valorisé, qu'il a dans l'espace public une image plutôt positive est déjà à mon avis une priorité que je suivrais.

Et, enfin, c'est toute la question des pratiques, c'est-à-dire, comment aujourd'hui on va permettre qu'il existe une véritable praxis et qu'un certain nombre de personnes deviennent des savants de leur propre réalité, que ces personnes produisent le sens dans l'action et que ce sens-là soit immédiatement réinjecté dans l'action en train de se faire.

Ce sont vraiment les deux priorités, car aujourd'hui je pense que, plus que jamais, les compétences professionnelles sont mises à l'épreuve et que l'on s'interroge sur ce qu'on peut encore apporter à une personne en situation de handicap ou en grande difficulté sociale. 